\title{
Que disent les locutions de, devant, et, en tant que, entre...?
}

Jean-Claude Gens

\section{(2) OpenEdition}

1 Journals

Édition électronique

URL : http://journals.openedition.org/alter/1386

DOI : 10.4000/alter.1386

ISSN : 2558-7927

Éditeur :

Association ALTER, Archives Husserl (CNRS-UMR 8547)

\section{Édition imprimée}

Date de publication : 1 octobre 2011

Pagination : 107-122

ISBN : 978-2-9522374-7-5

ISSN : $1249-8947$

\section{Référence électronique}

Jean-Claude Gens, «Que disent les locutions de, devant, et, en tant que, entre... ? », Alter [En ligne], 19 | 2011, mis en ligne le 01 janvier 2020, consulté le 23 janvier 2020. URL : http://

journals.openedition.org/alter/1386 ; DOI : 10.4000/alter.1386 


\section{QUE DISENT LES LOCUTIONS DE, DEVANT, ET, EN TANT QUE, ENTRE...?}

Jean-Claude Gens

Comme tous les termes d'une langue, les concepts philosophiques n'ont de sens que par leur relation à l'ensemble de la conceptualité à laquelle ils appartiennent. Mais cela signifie que la relation entre les concepts n' est pas moins essentielle que ces derniers. Et c'est cette relation que disent les éléments du langage que l'on appelle communément des particules de liaison comme de, et, après..., en dehors desquelles la parole serait pure nomination. Seulement, poser l'égale importance de ces particules par rapport aux termes qu'elles relient implique la mise en question du primat accordé par la logique aux concepts, et donc le primat ontologique des essences qu'ils désignent, c'est-à-dire le privilège accordé à ce que la tradition philosophique appelle les catégorèmes par rapport aux syncatégorèmes, ou encore aux énoncés auto-sémantiques ou auto-signifiants par rapport à ceux qui ne sont que co-sémantiques ou co-signifiants, et dont la diversite est irréductible aux connecteurs logiques.

Si cette distinction entre catégorèmes et syncatégorèmes est aujourd'hui interrogée par la sémantique prépositionnelle, il n'en reste pas moins que, en dehors de la tradition logique, l'interrogation du statut et de la fonction de ces particules constitue jusqu'au $20^{\text {e }}$ siècle un impensé de la réflexion classique sur le langage. Avant de considérer la transformation qui a, depuis, affecté la considération de ces particules, il convient néanmoins d'examiner l'horizon dans le cadre duquel celle-ci s'est jusqu'alors déployée, puis la manière dont deux philosophies du 19e siècle transforment cet horizon en ouvrant la voie à celles qui, au siècle suivant, achèveront d'intégrer les particules à la conceptualité philosophique.

Considérées isolément, l'inconsistance des particules tient au fait qu'elles ne comptent même pas comme des " parties » à part entière du discours, et ne «disent » donc pas grand-chose; si elles semblent vivre de la signification des catégorèmes et n'en n'être pour ainsi dire 
que les parasites, Robert Desnos ou Beaumarchais en ont néanmoins pointé le caractère décisif: Desnos, par exemple, dans son poème "Comme », qui, après avoir joué sur l'homonymie du comme, come, dans les langues européennes, dit: "Comme, je dis comme et tout se métamorphose, le marbre en eau, le ciel en orange, le vin en plaine, le fil en six, le cour en peine, la peur en seine " , Beaumarchais, dans la scène 15 de l'acte III du Mariage de Figaro où la discussion s'engage sur la question de savoir ce que signifie la promesse de Figaro a Marcelline de Verte-Allure: "Laquelle somme je lui rendrai et l'épouserai ». Que signifie ce et ? Faut-il l'entendre comme un et puis, ou comme un ou, voire un où, la controverse portant encore sur la question de savoir si la phrase doit s'entendre avec ou sans virgule... - une ponctuation à laquelle ont été particulièrement attentifs Nietzsche et Adorno $^{2}$. Le caractère déterminant des particules est patent au regard d'exemples plus simples encore si l'on admet que " être dans la rue » et "être à la rue », «dans la soupe!» et «à la soupe ! ", sont loin de signifier la même chose, c'est-à-dire que dans et et ne déterminent pas moins que rue et soupe la signification de l'énoncé.

Mais la première difficulté de leur considération tient au fait que ces syncatégorèmes sont grammaticalement et logiquement hétérogènes, puisque l'on a affaire à une jungle plus confondante que la " rhapsodie », comme disait Kant, des catégories aristotéliciennes. Comme on sait, la première de ces catégories correspond à un substantif et les quatre dernières ont un caractère verbal. Les autres catégories appartiennent en revanche aux syncatégorèmes, puisque ce sont des adjectifs indéfinis quantifié et qualifié (poson et poion), des adverbes indéfinis déterminants des circonstances, quelque part (pou) et à un moment (poté), et un syntagme adverbial indéfini, le relatif (pros ti). La difficulté est d'autant plus grande qu'un même syncatégorème, par exemple sur ou contre, peut fonctionner comme préfixe ou comme préposition, comme le rappelle Saussure, et comme préposition ou comme concept, dans des locutions comme envers et contre tout ou faire un contre.

Si Trendelenburg et Benveniste ont pu voir dans le caractère hétérogène des catégories aristotéliciennes l'expression de la diversité des classes linguistiques de la langue grecque, il est remarquable que cette disparité a suffisamment embarrasse les commentateurs de la tradition dominante pour qu'ils réifient ou substantialisent les adverbes et le syntagme adverbial en en faisant les catégories de lieu, de temps et de relation. Dans une direction opposee et au dire de Simplicius, des commentateurs anciens considéraient la liste des

\footnotetext{
1. Desnos, « Comme », in Les fortunes (1942), Paris, Gallimard, 1969.

2. Voir Adorno, «Signes de ponctuation » in Mots de l'étranger et autres essais, Paris, Éditions de la MSH, 2004.
} 
catégories comme incomplète en y pointant l'absence des conjonctions, des articles, des négations... On sait par ailleurs que si le terme même de syncatégorème n'est pas aristotélicien, bien que son usage en grec soit attesté à l'époque de Pricien, le Stagirite et ses commentateurs grecs distinguent déjà la signification de la consignification et de la sursignification ${ }^{3}$. Et, à côte des Sommes de logique à leur "heure de gloire», au XIII siècle, les syncatégorèmes se verront consacrer des traités spécifiques les dénombrant et discutant de leur signification ou de leur absence de signification, du mode affectif ou conceptuel de celle-ci... ${ }^{4}$ Plus récemment, le linguiste Krassimir Mantchev a élaboré un impressionnant "système prépositionnel du français » en cherchant à remonter aux premiers balbutiements de l'articulation de la pensée, mais c'est en excluant le genre des conjonctions comme « et $»^{5}$.

Ces analyses restent néanmoins exceptionnelles, et un passage du De magistro d'Augustin, relatif à la question de savoir de quoi le signe est un signe, me semble constituer un épisode plus représentatif du sort fait a ces particules dans l'histoire, qui, au fond, serait celle de leur refoulement. Partant du vers 652 du second livre de l'Enéide - Si nihil ex tanta Superis placet urbe relinqui - qui pose d'entrée de jeu la difficile question du statut de si, nihil, ex, Augustin prend l'exemple d'une forme aperçue dans le lointain et à propos de laquelle on pourrait dire : "Si c'est un homme, c'est un animal ». Si ne peut, en l'occurrence, se voir substituer correctement un parce que ; autrement dit, «le si convient» alors que «le parce que ne convient pas». $\grave{A}$ la question de savoir si «le si convient» et «le parce que ne convient pas » sont des propositions complètes, Adeodat répond par l'affirmative à Augustin. De façon à la fois compréhensible et étrange, ce dernier enferme alors la suite de l'analyse dans une remarquable alternative : "En ces propositions, quels sont les verbes et quels sont les noms? " Une fois repérés les verbes convient et disconvient, Augustin estime légitime de conclure que les «conjonctions » si et parce que sont donc des noms... ${ }^{6}$ Par ailleurs, lorsque ces particules se voient accorder une attention, c'est dans le cadre de considérations purement gnoséologiques, comme le montre encore l'exemple de Locke, qui est un des rares philosophes classiques à s'être penché sur elles.

\footnotetext{
${ }^{3}$. Voir Jean Lallot, «À propos des syncatégorèmes : consignification et signification adjacente dans la tradition logico-grammaticale grecque », Histoire épistémologie langage, vol. $\mathrm{n}^{\circ} 25$ fas. $\mathrm{n}^{\circ} 2$, 2003, p. 9-32.

${ }^{4}$. Voir Frédéric Goubier, "Les syncatégorèmes au XIII" siècle », in Histoire épistémologie langage, vol. $\mathrm{n}^{\circ} 25$ fas. no 2, 2003, p. 85-113.

5. K. Mantchev, La linguistique, CEuvres, tome 1, Paris, L'Harmattan, 2004, et J. Cervoni, La préposition. Etude sémantique et pragmatique, Paris - Louvain-la-Neuve, Duculot, 1991, p. 159-163.

${ }^{6}$. Saint Augustin, De magistro, trad. B. Jolibert, Le maître, Paris, Klincksieck, 2002, p. 50, et, plus largement, p. 46-50.
} 
L'Essai philosophique concernant l'entendement humain leur consacre ainsi le chapitre 7 du livre III, qui traite des «mots». Intitulé On particles, ce chapitre distingue en dehors des mots qui sont des noms pour les idées, ceux qu'il qualifie de particles et qui relient aussi bien des propositions que des idées entre elles - la liaison des idées étant, au fond, déjà patente dans la composition même des concepts classiques de sub-stance, de su-jet ou d'ob-jet, ou de con-cept... Si le premier exemple de Locke se réfère au jugement d'existence «cela est » qui témoigne d'une " affirmation » de l'esprit, la fin du chapitre met en évidence la complexité des différentes significations possibles de ces particules en prenant l'exemple de but. But dit ainsi aussi bien la réserve, la justification, le retour... Sauf que Locke précise encore que les connexions sont «données » par, ou sont le fait de l'esprit : ce sont des formes que l'esprit prend en discourant, des "marques de quelque action de l'esprit, ou de quelque chose qu'il veut donner à entendre », des marques qui ne suffisent pas à dire la multiplicité des "vues, postures, situations, tours [...] et autres pensées de l'esprit » $(\S 4)$, ou, dans les termes de Leibniz, des « opérations de l'entendement ", de sorte que chacune de ces particules en signifie plusieurs.

L'analyse lockienne a pour objet de déterminer l'art de bien penser et de bien communiquer. Autrement dit, sa visée est donc d'ordre logique et rhétorique, et elle ne va pas jusqu'à mettre en question la croyance en l'existence d'entités que Locke pense de manière atomiste. Cette dimension logique et gnoséologique est encore celle dans laquelle s'inscrivent les analyses de Brentano, de la quatrième des Recherches logiques IV de Husserl ${ }^{7}$ ou de Russell, comme, plus récemment, du Historisches Wörterbuch der Philosophie de Ritter. La phénoménologie va néanmoins articuler ses descriptions en jouant des subtilités de sens des particules en les intégrant dans sa conceptualité même, et en en déportant la signification sur le plan de l'existence et de l'éthique.

Le poids décisif des particules est en effet patent dès l'énoncé même du principe phénoménologique de l'intentionnalité de la conscience. Comme le remarque joliment Belaval : «Les deux petites lettres de la préposition "de" ont bouleversé la description des faits de conscience. L'expression "conscience de" interdit certaines affirmations et contraint d'en énoncer d'autres : elle est, à elle seule, une méthode de recherche $»^{8}$. La même chose vaut, inversement, de la particule pour, toute chose n'étant jamais que pour la conscience. La chose est plus évidente encore dans la phénoménologie déployée dans Etre et temps, dès l'explicitation du mode d'être du Dasein en tant

\footnotetext{
7. Husserl, Recherches logiques IV § 9, trad. H. Elie, A. Kelkel et R. Schérer, Paris, Presses Universitaires de France, 1993.

8. Yvon Belaval, Les philosophes et leur langage (1952), Paris, « TEL », Gallimard, 1990, p. 120.
} 
que in der Welt, une explicitation qui considère le là, et comprend aussi le est du "je suis» comme un "habiter», comme être-aumonde, à-la-mort, avec...

Ces particules ne sont pas co-signifiantes si l'on entend par là qu'elles co-déterminent secondairement des significations qui sont avant tout portées par des substantifs - ce que disent pourtant littéralement le terme grammatical d' «adverbe » et celui, logique, de «syncatégorème ». C'est la raison pour laquelle ce terme demande à se voir substituer celui de "particule ", qui, lui, n'implique pas de souscrire au primat du « catégorème ». A la différence des catégories, les particules $n$ 'indiquent par ailleurs ni des contenus représentatifs ou des essences, ni des marques ou des opérations de l'esprit - un quoi? ou un que? -, mais différents modes intentionnels de la conscience, différentes modalités de la donation des phénomènes, le comment du donné : la donation sur le mode d'un avec, d'un pour, d'un sans, d'un et... Reprenant explicitement une suggestion de Bergson, Merleau-Ponty dit ainsi l'appartenance au monde en termes $d^{\prime} u n$ " j'en suis $»^{9}-$ une expression que l'on pourrait aussi entendre comme une traduction de l'habiter heideggérien. «En » être, ce n'est pas être $d e$, dans ou pour. De manière comparable, Binswanger forge, pour penser une modalité spécifique de l'être-avec, l'expression de prendre-par, de Nehmen bei.

Mais l'attention heideggérienne accordée à la façon dont la pensée et ce qu'elle pense s'articulent grâce aux particules s'atteste déjà dès le début des années vingt, par exemple, dans le cours du semestre d'hiver consacré aux Phänomenologischen Interpretationen zu Aristoteles (Interprétations phénoménologiques d'Aristote) de 1921-1922. Cherchant à dégager les " catégories fondamentales de la vie », la troisième partie de ce cours invite ainsi à découvrir le phénomène de la vie comme s'articulant "dans, de, à partir de, pour, avec, et contre un monde » («in, aus, für, mit und gegen eine Welt») ${ }^{10}$. Et ceci vaut encore des écrits plus tardifs comme Le principe de raison dont la lecture du vers d'Angélus Silésius « La rose fleurit sans pourquoi » interroge le sens de weil.

Il convient pourtant de remarquer que cette attention de Heidegger aux particules et le plan sur lequel il les mobilise s'inscrit dans la continuité de la pensée kierkegaardienne, dans la mesure où les concepts qu'il appelle indicatifs ou assignations formelles disent non

\footnotetext{
9. Merleau-Ponty, Eloge de la philosophie, Paris, Gallimard, 1975, p. 23, et Le visible et l'invisible, Paris, Gallimard, « TEL », 1990, p. 164, 169.

10. Heidegger, Gesamtausgabe 61 - désormais cité GA -, Francfort-sur-le-Main, Klostermann, 1985, p. 85 sq. ; dans le même sens, voir par exemple GA 34, 14, trad. A. Boutot, De l'essence de la vérité : "La parole d'Héraclite porte à la parole cette expérience fondamentale avec, dans et à partir de laquelle s'est éveillé un regard sur l'essence de la vérité comme hors-retrait de l'étant », Paris, Gallimard, 2001, p. 30.
} 
des choses ou des entités, mais des directions dans lesquelles est susceptible de se déployer l'existence. Les Phänomenologischen Interpretationen zu Aristoteles répondent ainsi comme en écho à la manière dont $\mathrm{Ou}$ bien... ou bien détermine le propre de la vie esthétique : "Celui qui vit dans, par, de et pour l'esthétique qui est en lui, vit sur le plan esthétique $»^{11}$.

Seulement, si la diversité de ces particules nomme pour Kierkegaard les différents aspects sous lesquels demande à être entendue une telle vie, les différents stades de la vie et leurs catégories sont eux-mêmes saisis de manière plus radicale par certaines particules. Autrement dit, à la différence de celles qui sont considérées sur le seul plan logique ou gnoséologique, ces catégories et les particules qui en ramassent le sens correspondent à des attitudes ou des modes d'être qui se situent à des niveaux différents. Pour commencer par la plus célèbre d'entre elles, la catégorie kierkegaardienne de la subjectivité ne se comprend pas, si ce n'est à partir de devant dont on connaît la centralité dans la pensée de Luther pour lequel coram mundo et coram deo disent les deux possibilités antithetiques de l'existence ${ }^{12}$.

La première partie de La maladie à la mort commence ainsi par mettre en évidence le fait que la subjectivité est un rapport se rapportant à lui-même, pour autant qu'il se rapporte à ce qui a posé ce rapport - ce qui est, en termes plus aristotéliciens, une façon de penser le caractère non substantiel, mais relationnel de la subjectivité. Mais cet être relatif à signifie surtout, précise le début de la seconde partie de La maladie à la mort, que la subjectivité n'est et ne se comprend elle-même que devant Dieu ${ }^{13}$ - aucun des deux termes de cette locution n'étant plus essentiel que l'autre. Autrement dit, la nature du moi est déterminée par ce devant quoi il est un moi, " devant les vaches », " devant des esclaves », « devant l'État », si ce ne sont que des mois respectivement de bouvier, de souverain ou de bourgeois ${ }^{14}$, ou devant Dieu, un devant qui, seul, donne son sens au péche. On connaît par ailleurs la postérité du thème chez Lévinas et Ricoeur invitant à une compréhension forensique du texte, pour autant que comprendre, c'est se comprendre devant le texte. De telles particules sont donc des catégories de l'existence, ou, en termes heideggériens, des existentiaux.

\footnotetext{
11. Kierkegaard, Ou bien... ou bien, trad. P.-H. Tisseau et E.-M. Jacquet-Tisseau, L'alternative, CEuvres complètes, Paris, Éd. de L'Orante (désormais cité OC suivi du tome et de la page), vol. 4, 1970, p. 162, rééd. R. Laffont, « Bouquins », 1993, p. 516.

12. Voir G. Ebeling, Luther. Einführung in seinem Denken, Tübingen, Mohr Siebeck, 1964, p. 220 sq. 13. Sur devant, voir encore par exemple Post-scriptum aux miettes philosophiques, OC 11, 159 sq., ou trad. P. Petit, Paris, Gallimard, TEL, 2002, p. 392 sq., et «Des pensées qui attaquent dans le dos », OC 15, 159, 162, 175 et 177.

14. Kierkegaard, La maladie à la mort, OC 16, 235, ou « Bouquins », p. 1261.
} 
Il en va de même de ces deux autres catégories subjectives que sont le choix et l'instant. Ces derniers peuvent bien en tant que substantifs donner lieu à des analyses intellectuelles ou d'entendement. En revanche, exprimé de manière plus incisive et existentielle, les véritables noms de ces catégories sont ou bien... ou bien, qui est une conjonction disjonctive ${ }^{15}$, et les particules quand, lorsque ou maintenant, et si, dont les Discours chrétiens de 1848 interrogent la signification, en particulier dans les commentaires des versets 8, 28 et 13, 11 de l'Épître aux Romains. Se référant à l'art socratique d'interroger, le commentaire du verset « Nous sommes maintenant plus proches du salut - que lorsque nous avons cru » $(\mathrm{Rm} 13,11)$ demande :

"Es-tu donc "maintenant" plus proche du salut? - Oui. - Mais plus proche que quand? »Ici peut-être se produit un arrêt qui, à ce point, risque de plonger tout le discours dans la confusion. Tu es maintenant "plus proche»; ce "plus proche» est une comparaison, mais à quoi l'appliques-tu? Peut-on dire qu'un homme est plus grand qu'un autre qui n'existe pas? Il y a quelque chose dans ce "plus» de la comparaison quelque chose de tentant, de persuasif qui vous attire, comme si les choses allaient d'elles-mêmes, comme si l'on ne devait pas se défier ${ }^{16}$.

Et s'il faut s'en défier, c'est que de telles pensées peuvent non seulement être un sujet de consolation, mais «en quelque sorte attaquer dans le dos », puisque c'est dans l'effroi du péril et non dans la tranquille assurance d'une plus grande proximité que croît ce qui sauve ${ }^{17}$. Maintenant et lorsque ne remplissent donc pas la fonction d'adverbes circonstanciels, mais posent des questions, ou encore, comme le précise Kierkegaard à propos de ou bien... ou bien, ce sont des interjections $»^{18}$. Il me semble inversement évident que le car, le et ou le mais n'en sont pas, c'est-à-dire ne disent pas la subjectivité.

Comme les particules qui articulent des argumentations, le car dans la mesure où il dirait le sérieux d'une vie qui ne peut plus se vivre dans la dimension esthétique sans pour autant s'engager véritablement dans la vie éthique - un car que l'on pourrait encore

\footnotetext{
15. La traduction de Enten - Eller par L'alternative intellectualise à cet égard la pensée de Kierkegaard, elle réifie une conjonction disjonctive et manque la tournure usuelle de ou bien... ou bien à laquelle ce dernier recourt communément, par exemple OC 4, 145 et 151 ("Ou bien coiffeur - ou bien comptable de banque »), ou «Bouquins », p. 501 et 506 ; voir encore, dans le même sens : «Ces mots de ou bien... ou bien sont un petit poignard à double tranchant que je porte sur moi et grâce auquel je puis donner par surprise le coup de grâce à toute la réalité [...] », Pap. III B 179, cité par Régis Boyer dans sa préface aux œuvres de Kierkegaard publiées par R. Laffont, in «Bouquins », p. 5.

16. «Des pensées qui attaquent dans le dos », in OC 15, 207.

17. « Des pensées qui attaquent dans le dos », in OC 15, 208.

18. Ou bien... ou bien, OC 4, 145, ou «Bouquins», p. 501. La dimension interrogative de cette structure disjonctive - conjonctive s'atteste encore dans le titre Coupable? - Non coupable?
} 
interroger à partir de la lecture de weil par Le principe de raison. Le et, dans la mesure où il dirait le mode d'être de la vie esthétique qui se perd dans la multiplicité de l'immédiat et se refuse à choisir, à se choisir - le mode d'être, par exemple, de Don Juan. C'est du moins dans ce cadre que le Post-scriptum s'arrête à d'autres particules comme mais, si et au moins. Considérant la décision avec laquelle commence l'existence éthique, Kierkegaard en pointe l'incompatibilité avec tout compromis relatif à ce qui peut avoir une "signification historico-mondiale » à laquelle s'intéressent les hommes et les philosophes. Ainsi,

celui qui prend la décision dit: je veux - mais alors je veux en même temps avoir une importance dans l'histoire mondiale - "aber » [mais]. Il y a donc un "aber" - ainsi disparaît de nouveau l'Esprit [...], et le commencement n'a pas lieu [...]. La moindre trace d'un aber et le commencement est faussé19.

Kierkegaard s'arrête alors à l'exercice du pouvoir qui est peut-être ce qui fascine le plus sur le plan historico-mondial, d'une histoire mondiale devant laquelle "l'éthique se sent intimidée comme un moineau dans une danse de hérons $»^{20}$ :

Si un roi disait à ses ennemis: faites ce que j'ordonne, ou, sans cela, tremblez devant mon sceptre qui s'abattra sur vous d'une façon terribleà moins qu'il ne plaise à la Providence de me prendre aujourd'hui même mon trône et de faire d'un gardien de pourceaux mon successeur! Pourquoi entend-t-on si rarement ce si et cet à moins que, cette dernière partie du discours qui est la vérité éthique? ${ }^{21}$.

Ce sont, inversement, les articulations d'un discours anonyme se déployant dans la sphère des généralités, en laquelle se réfugie l'entendement, que récapitule à sa façon le fameux mais où est donc Ornicar? des écoliers. De telles considérations n'impliquent pourtant pas que la signification de telle ou telle particule serait univoque en tant qu'elle renverrait toujours à un mode de discours donné et au stade correspondant de l'existence. L'équivocité des particules rappelée par Locke à propos de but est au contraire demultipliée puisqu'une particule signifie également en fonction du stade à partir duquel elle est considérée, c'est-à-dire aussi interrogée - ou non interrogée ${ }^{22}$. Il n'en reste pas moins que certaines d'entre elles sont susceptibles de faire l'objet d'une interrogation spécifique et ainsi de

\footnotetext{
19. Post-scriptum aux miettes philosophiques, OC 10, 130, ou trad. P. Petit, op. cit., p. 125.

${ }^{20}$. Post-scriptum, OC 10, 133, ou trad. P. Petit, dont nous suivons ici la traduction, op. cit., p. 127.

21. Post-scriptum, OC 10, 132, ou trad. P. Petit, op. cit., p. 126.

22. Sur les différentes acceptions du si, voir par exemple «Des pensées qui attaquent dans le dos », in OC 15, 225 et 228.
} 
signer une pensée donnée, de la distinguer par l'espace ou la dimension ouverte par une telle interrogation. Devant signe ainsi la pensée de Kierkegaard ou une pensée d'inspiration kierkegaardienne.

Kierkegaard me semble entreprendre une révision de la théorie aristotélicienne plus radicale que celle de la Critique de la raison pure. $\mathrm{D}^{\prime}$ abord en invitant à penser les catégories comme fondamentalement "de » l'existence, ensuite en élevant à la dignité du concept les particules, et enfin en ne succombant pas à la tentation d'en élaborer une «table » dans ce qu' elle peut avoir de systématique - un tableau "bien compartimenté", comme ceux que se feraient du monde certains « instituteurs hirsutes ou conseillers en retraite auprès la cour d'un tribunal régional $\aleph^{23}$. A cet égard, on peut se demander si les formes a priori de l'expérience dégagées par la Critique de la raison pure ne résultent pas d'une réification des particules ici, là-bas, contre, maintenant... en ce qui concerne celles de l'intuition, et à, tout, quelque, car, si... alors, toujours..., en ce qui concerne celles de l'entendement. Dans une telle perspective, les considérations des particules se départageraient entre celles qui sont pré- et celles qui post-kierkegaardiennes. Même s'il ne semble pas l'avoir lu, un autre philosophe du $19^{\mathrm{e}}$ siècle a accordé une attention encore plus explicite aux particules et en a renouvelé la compréhension dans une perspective qui, comme chez Kierkegaard, déborde largement les considérations d'ordre logique et linguistique, voire leur est radicalement étrangère : William James. Ce dernier suggère en effet que le privilège accordé à telles ou telles particules determine obscurément la singularité, c' està-dire le style, de chaque philosophie.

De manière à première vue étonnante, James reconduit les différentes façons dont les philosophies rendent compte de l'expérience à des différences dans l'accentuation, ou l'occultation, de telles ou telles dimensions expériencielles exprimées par les particules. «Un monde d'expérience pure » avance ainsi que "la philosophie s'est toujours jouée sur les particules grammaticales ${ }^{24}$. Une telle considération des particules repose sur la thèse - explicitement critique à l'égard de Hume - selon laquelle les relations entre les choses, qui nous sont données dans l'expérience, ne sont pas moins réelles que les choses qu'elles mettent en relation. Exprimees par des particules comme est, puis, avant, dans, sur, entre, pareil..., ces relations appartiennent à l'expérience au même titre que les sujets et les prédicats, les noms et

\footnotetext{
23. Heidegger, GA 42, 46, trad. J.-F. Courtine, Schelling, Paris, Gallimard, 1977, p. 55.

24. Essais d'empirisme radical, trad. G. Garetta et M. Girel - désormais cité EER -, Marseille, Agone, 2005, p. 60. Pour une présentation plus détaillée de la perspective jamesienne, je me permets de renvoyer à L'expérience vive, Paris, Presses Universitaires de France, 2009, p. 92-99, dont les lignes ci-dessous sont une version abrégée.
} 
les adjectifs. L'empirisme défendu par James, et qu'il qualifie de radical, prolonge The Principles of Psychology qui avançaient déjà :

Il n'y a pas une conjonction ou une préposition, et pratiquement pas une phrase adverbiale, une forme syntaxique, ou une inflexion de la voix, dans le discours humain, qui n'exprime pas une nuance quelconque de relation que, à un moment donné, nous sentons actuellement exister entre les objets plus amples de notre esprit [...]. On pourrait dire un sentiment de et, de si ou de mais, aussi simplement que nous disons un sentiment de bleu ou de froid ${ }^{25}$.

S'il faut ainsi reconnaître une dimension affective à la signification de ces particules, la Stimmung que chacune d'elles est susceptible d'éveiller varie corrélativement à ses diverses acceptions. En termes kierkegaardiens, il serait nécessaire de reconnaître à ces particules ce que Le concept d'angoisse appelle, en ce qui concerne les concepts au sens usuel, une « atmosphère $»^{26}$.

Percevoir les relations dites par les particules, c'est en second lieu percevoir, par-delà sa discrétion, la continuité de l'expérience. Pour marquer cet être relationnel ou corrélatif de toute chose, James accorde un privilège à la " conjonction » au sens large de ce terme qui désigne tous les modes de co-ordination, y compris ce que l'on appelle la disjonction, puisque celle-ci dit une relation entre les termes disjoints. Et en tant qu'elle s'inscrit dans un devenir, cette liaison ou conjonction dit l'ensemble de la réalité comme jeu de conjonctions et de disjonctions se faisant et se défaisant. Cela signifie encore que les relations sont des transitions, faute de quoi elles seraient pensées comme des entités intemporelles: "La vie se trouve dans les transitions tout autant que dans termes reliés » (EER, 84). Ces transitions disent ainsi la croissance, par addition, de l'univers, et, de ce fait, de la connaissance qu'il nous est possible d'en avoir. Comment entendre maintenant la these selon laquelle la philosophie s'est toujours jouée sur la question des particules?

Si l'on admet que la manière dont les philosophies ont pensé l'expérience relève soit de l'empirisme soit du rationalisme, le premier considère au fond les données de l'expérience comme absolument disjointes, alors que le second reconduit la diversité de l'expérience à l'unité fictive d'un absolu ou d'une substance. À la "pulvérisation générale de l'expérience entière par l'association » des empiristes classiques répond la correction « artificielle » du rationalisme

\footnotetext{
25. James, The Principles of Psychology, New York, Holt, 1918, p. 245 (souligné par James).

26. Voir, dans le même sens, Wittgenstein, par exemple, Recherches philosophiques, trad. F. Dastur, M. Elie, J.-L. Gautero, D. Janicaud et E. Rigal, Paris, Gallimard, 2004, p. 258 sq., à propos du si et de son atmosphère, Le cahier bleu et le cahier brun, trad. G. Durand, Paris, Gallimard, "TEL », 1998, p. 164, ou Remarques sur la philosophie de la psychologie I, trad. G. Granel, Mauvezin, TER, $1989, \S 331-335$, p. 84 .
} 
qui lui ajoute des "agents d'unification trans-expérientiels [...], des substances, des facultes intellectuelles, ou encore des Sois » (EER, 59).

De manière plus concise, cela signifie que la ligne de rupture entre l'empirisme et le rationalisme traditionnels tient à leur sous- ou surestimation des particules avec (EER, 61) ou et, aussi, et plus, en tant qu'elles disent une pure addition, c'est-à-dire le caractère inachevé de l'expérience, alors que car, donc, ainsi, tout..., prêtent une cohérence au monde, qui est loin de l'expérience que nous en avons. La relation conjonctive, sous la pluralité de ses modes, permet en revanche de penser la co-ordination ou la cohésion d'un divers, c'est-à-dire à la fois l'unité et la séparation, et on comprend que James écrive :

Être un empiriste radical, c'est défendre cô̂te que coûte cette relation conjonctive entre toutes, car c'est là le point stratégique, la position par laquelle, si une brèche était pratiquée, toutes les corruptions de la dialectique et toutes les fictions métaphysiques se déverseraient dans notre philosophie (EER, 62).

Il est dès lors possible d'ordonner les particules - précédemment énumérées de manière arbitraire - selon le degré croissant « $d$ 'intimité ou d'inclusion » de la conjonction qu'elles désignent: de avec, près de... jusqu'à à travers et mon (EER, 60). Le chapitre cinq du Pragmatisme propose ainsi des exemples concrets d'expérience de ces différents degrés de relations ou de "conjonctions »: entre ma vie psychique et celle des autres, entre ma vie psychique et la vie organique de mon corps, et entre les diverses tendances de ma propre vie psychique $^{27}$.

La prétention de ces analyses jamesiennes est donc ontologique au sens classique de ce terme si l'on conçoit l'être de ce qui est comme essentiellement fluant. Seulement, cette dimension est néanmoins seconde au regard de ce qui importe finalement à James :

«La question réellement vitale pour nous tous est Que va devenir ce monde? Qu'est-ce que la vie est susceptible de faire d'elle-même? Le centre de gravité de la philosophie doit par conséquent se déplacer $\gg(P, 169)$.

Autrement dit encore, la question n'est pas de savoir quel est l'être des choses, mais la direction vers laquelle celles-ci sont susceptibles de se diriger et de se déployer. Et, à cet égard, ce seraient les particules et puis, et après, vers..., qui diraient l'expérience en tant qu'elle est grosse d'un à venir. L'horizon commun de l'attention accordée aux particules par Kierkegaard et James est donc d'ordre éthique, même si la manière dont ce dernier est pensé diffère 27. James, Le pragmatisme (1907), trad. N. Ferron - désormais cité «P» suivi de la pagination
dans le corps du texte -, Paris, Flammarion, «Champs », 2007, p. 194. 
considérablement, et, comme cela a déjà été suggéré à propos de Heidegger, une telle interrogation ouvre la voie à une reconsidération de ces particules au siècle suivant.

Si l'on admet que la pensée philosophique se joue, c'est-à-dire se déploie dans un espace qui lui est accordé par de, pour, devant, après, avec..., cet espace de jeu ne se réduit pas au blanc, au vide silencieux, entre les mots, mais est peuplé par le murmure de la jungle de ces particules. Seulement, certaines d'entre elles peuvent prétendre à une reconnaissance insigne, et la pensée se joue alors en un second sens par le privilège clairement ou obscurément accordé à certaines particules plutôt qu'à d'autres, par exemple lorsqu'elle se décide pour la copule est, ou car, ou, au contraire, joue le et contre le est. Ainsi, la singularité de la pensée kantienne ne tiendrait pas tant à l'élaboration d'une table des catégories qui participerait de l'oubli des particules propre à la tradition logique dominante, qu'au als $o b$, qui s'inscrit dans le sillage de la distinction entre connaître et penser pour habiter les diverses dimensions de la pensée critique, et que Kant aurait fini par considérer comme le point de vue le plus élevé de la philosophie transcendantale.

Parmi la jungle des particules, als ob semble néanmoins difficilement pouvoir prétendre au statut d'archi-particule, face au als ou à l'en tant que et, plus encore, au "débonnaire » et, comme dit Heidegger ${ }^{28}$. Ce privilège semble flagrant chez Deleuze et Derrida. Ironisant sur la hantise de la langue allemande par la nostalgie de l'être "qui fait tendre vers lui toutes les conjonctions dont [l'allemand] se sert pour fabriquer un mot composé ", et renvoyant à Russell et à Whitehead, Deleuze invite à «substituer le et au est. A et B. Le et n'est même pas une relation ou une conjonction particulière, il est ce qui sous-tend toutes les relations ${ }^{29}$. Et s'il pense l'unité minimale en termes $\mathrm{d}^{\prime}$ " agencement», ce qui le distingue stratégiquement de James qui engloutit le est dans une rhapsodie de particules, c'est la substitution pure et simple du et au est:

Tout n'est que rencontre dans l'univers, bonne ou mauvaise rencontre. [...] Adam mange la pomme, le fruit défendu ? C'est un phénomène du type indigestion, intoxication, empoisonnement: cette pomme pourrie décompose le rapport d'Adam ${ }^{30}$.

\footnotetext{
28. Heidegger, GA 41, 16, trad. J. Reboul et J. Taminiaux, Qu'est-ce qu'une chose ?, Paris, Gallimard, 1971, p. 28.

29. Gilles Deleuze - Claire Parnet, Dialogues, Paris, Flammarion, «Champs », 1996, respectivement p. 73 et 71 .

${ }^{30}$ Gilles Deleuze - Claire Parnet, Dialogues, op. cit., p. 74.
} 
Cela reviendrait à reconsidérer et réévaluer la catégorie aristotélicienne du relatif, qui est à la fois la seule à se voir effectivement définie par les Catégories et celle dont le propre ne peut se dire que par rapport à un autre. Si Aristote pouvait ainsi la penser comme la plus éloignée, par nature, de l'ousia ou de l'essence singulière ${ }^{31}$, Trendelenburg invitait à l'expulser de la liste. Mais la question est justement de savoir si l'être de ce qui est n'est pas essentiellement relationnel, ou, mieux, si les particules ne disent pas l'être relationnel et donc mobile des choses, un être relationnel au regard duquel l'être substantiel est fictif. À ce titre, et serait la catégorie des catégories, celle, au fond, que déclineraient les autres particules, et c'est finalement la notion même de relation qui demanderait à être récusée, pour autant qu'elle maintient ou présuppose encore la différence, ou plutôt la consistance, des termes reliés.

À cet égard, dans son remarquable Et cetera, Derrida commence par mettre en évidence la manière dont le et, qu'il qualifie de « quasitranscendantal », est " silencieusement impliqué dans toute liaison ou conjonction syncatégorématique » - un et " sans lequel rien n'arriverait, ni l'enchaînement, ni la rupture, [...], ni l'être-avec, ni l'être-sans, ni l'être-ensemble ${ }^{32}$. Mais c'est finalement la ligne même de partage entre catégorèmes et syncatégorème qui s'évanouit dans la mesure où "la déconstruction introduit un "et" $\mathrm{d}$ 'association et de dissociation au cœur même de chaque chose, elle reconnaît plutôt cette division de soi au-dedans de chaque concept. [...] Il y a écriture et écriture, invention et invention, don et don ${ }^{33}$. Ce et pointe donc la dimension à l'intérieur de laquelle quelque chose est susceptible de se donner en tant que telle - une dimension qui rend aussi possible un avec et un ensemble. Il est dès lors permis de se demander si ce n'est pas dans le cadre du comme ou de l'en tant que que se déploie le discours philosophique.

S'inscrivant dans la continuité de Heidegger, dont les Concepts fondamentaux de la métaphysique considèrent précisément la structure de l'en tant que comme la « condition de possibilité du logos », Deleuze avance ainsi que la philosophie est «l'art de l'en tant que ${ }^{34}$. Dans la mesure où il « rend explicite l'étant en tant qu'il est constitué de telle ou telle manière », l'en tant que signifie une tâche, précise Heidegger, et, à ce titre, c'est une assignation formelle ${ }^{35}$. L'en tant que désigne

\footnotetext{
31. Aristote, Métaphysique, N, 1, 1088 a 22 sq.

32. Derrida, «Et cetera », in Derrida, Cahier de L'Herne, Paris, 2000, rééd. Et cetera, Paris, Carnets de L'Herne, 2005, p. 37 et 55.

33. Et cetera, op. cit., p. 8 sq.

34. « Ce n'est pas par hasard que la philosophie emploie constamment un mot qu' on lui reproche [...], c'est l'espèce de locution "en tant que" [. La philosophie, c'est] l'art de l'en tant que ", Cours de Vincennes (1978-1981) sur Spinoza.

35. GA 29-30, trad. D. Panis, Les concepts fondamentaux de la métaphysique, Paris, Gallimard, 1992, $\S 69-72$.
} 
«le moment structurel de cet entre-deux (ou de cet entre - des Zwischens) qui fait originellement irruption » et qui constitue la dimension à l'intérieur de laquelle quelque chose est susceptible d'apparaître ${ }^{36}$.

Cet en tant que ne saurait donc être confondu avec celui qui caractérise l'appréhension d'un étant qui se donne sous tel ou tel profil, comme doué de telle ou telle signification. Car une telle appréhension est aussi celle de l'animal pour lequel la différence entre l'être et l'étant n'a pas de sens, puisqu'il appréhende bien une chose comme abri ou comme proie, mais pas en tant que telle, de sorte qu'il pourrait l'interroger quant à son être. Là où la langue allemande n'a qu'un simple als, le français permet ici de différencier un tel comme de l'en tant que. Et c'est à partir de ce dernier que Heidegger invite à penser le déploiement des ou bien... ou bien, ainsi ou comme, etc... Ainsi, pour autant que le projet, la projection, " dévoile l'être de l'étant » :

L'éclair de lumière (das Lichtblick) qui rend visible le possible ouvre celui qui projette à la dimension $d u$ "ou bien... ou bien», du "non seulement... mais encore » [ce qui est une modalité du et], de l'« ainsi » et de $l^{\prime}$ « autrement », du «quoi », du «est » et "n'est pas». Ce n'est que dans la mesure où cette irruption a eu lieu que deviennent possibles le "oui» et le "non", ainsi que le fait de questionner. La projection met en suspens dans la dimension du possible en général, et ainsi la décèle - un possible qui est déjà articulé dans le possible de l'«être ainsi et autrement », de "la possibilité et de l'impossibilité d'être»(des Ob- und des Ob-nicht-seins) ${ }^{37}$.

L'ouverture de cet espace est donc ce que dit encore plus clairement la particule entre, qui n'est pas plus univoque que et, comme suffisent à le montrer des locutions comme "entre Berlin et Prague », " entre la poire et le fromage ", où entre dit aussi bien une approximation ou une comparaison, qu'une distance susceptible d'être parcourue, un possible itinéraire. Mais, en tant qu'il ouvre un espace, entre dit un interstice, un moment dont la consistance reste indéterminée, comme «entre chien et loup » qui désigne non pas une relation entre deux termes distincts, mais une dimension anterieure à leur différenciation, le vide, çûnyatâ, au sein duquel un différend, c'est-à-dire la multiplicité des particules, est susceptible de se déployer ${ }^{38}$. Cet entre, qui n'est assurément pas une « pré-position » ou

\footnotetext{
36. GA 29-30, 531, trad. Les concepts fondamentaux de la métaphysique, op. cit., p. 524.

37. GA 29-30, 530, trad. Les concepts fondamentaux de la métaphysique, op. cit., p. 523 sq.

38. Voir Heidegger, GA 13, 204-210, trad. F. Fédier et J. Beaufret, «L'art et l'espace », in Questions $I V$, Paris, Gallimard, 1976, p. 98-106, et "La chose ", GA 7, 171, trad. in Essais et conférences, Paris, Gallimard, 1958, p. 200. Sur ce point, je me permets de renvoyer à « L'inspiration extrêmeorientale de la méditation heideggérienne de l'espace", in Esthétiques de l'espace. Occident et Orient, J.-J. Wunenburger et V. Tirloni éd., Paris, Mimesis, 2010, p. 131-147.
} 
un «mot de liaison ", c'est aussi celui dont Fink esquisse encore le sens dans le séminaire avec Heidegger sur Héraclite en déclarant: "L'homme se rapporte à la fois à l'ouvert et à l'obscurité qui cache ", $c^{\prime}$ est l'homme "en tant qu'il est "l'entre nuit et jour" »39. En un sens certes différent, c'est encore l'entre que dit aida chez Bin Kimura, et que Buber considérait comme "l'archi-catégorie de la réalité humaine ». Et, à cet égard, la particule demande a être entendue non pas comme participant $\mathrm{d}$ 'une signification qui lui est intrinsèquement étrangère, mais à partir de parturire qui a donné la parturiente, puisque c'est de la particule que s'engendrent ou se déploient les significations des «parties» du discours. Mais pour autant que dire cet entre suppose de recourir à l'en tant que et au et, il faudrait ici convenir, à propos des particules, de ce que Aubenque dit des catégories : elles s'entre-signifient constamment ${ }^{40}$ - ce qui relativise aussi la quête d'une particule qui serait « la » plus originaire.

Dans une perspective plus éloignée de la phénoménologie, Michel Serres déclare néanmoins de maniere comparable viser « un transcendantal des relations » en invitant à penser « le long des particules $»^{41}$. L'incandescent avance ainsi que la réponse à la question Qui suis-je? en termes d'être-avec ou d'être-pour demande à se voir substituer les pures prépositions qui suffisent à créer « le langage, son sens et le sujet qui parle ». Explorant les variations de la langue française autour de la racine vers, Serres esquisse encore comme une grammaire des particules : "Le génitif et le datif, de, avec et à, éclairent, par exemple, les situations parasitaires, et l'ablatif, par, à partir de, peut dater des fondations $»^{42}$. Dans une telle perspective, la seule parole qui arriverait à se passer en quelque maniere de ces particules, ce serait celle non de la pure nomination, mais celle qui se déploie dans la parataxe et la tautologie auxquelles recourent parfois Serres et Heidegger.

La singularité de l'attention accordée aux particules par Kierkegaard, James et les figures de la philosophie du $20^{\mathrm{e}}$ siècle, dont il a précédemment été question, c'est d'avoir mis en question la distinction traditionnelle entre catégorème et syncatégorème. Les particules relèvent en effet du conceptuel, pour autant que le sens du « concept » est toujours transi de celui que celles-ci lui confèrent. En d'autres termes, les concepts au sens propre, c'est-à-dire usuel, sujets et prédicats, ou les mots, noms et verbes, ne sont donc pas moins en

\footnotetext{
39. Heidegger, GA 15, 211, 213 sq., trad. J. Launay, P. Lévy, Héraclite, Paris, Gallimard, 1973, p. 181 et 182.

40. P. Aubenque, Le problème de l'être chez Aristote, Paris, Presses Universitaires de France, 1972, p. 195.

${ }^{41}$. M. Serres, Éclaircissements, Paris, Champs, Flammarion, 1994, p. 172.

42. M. Serres, L'incandescent, Paris, Éditions Le Pommier, 2003, p. 265 et 273.
} 
attente des particules pour pouvoir signifier, que les particules des concepts ou des mots. Mais si - pour le dire dans les termes de la distinction de Fink entre concepts thématiques et opérants - ces philosophies ont pris pour thème ces particules opérantes, leur spécificité est d'avoir déplacé le champ de leur considération du logique et du sémantique à l'éthique et à l'ontologique. Dès lors, le trait distinctif du style de l'existence ou de la pensée se dirait selon les archi-particules qui, plus ou moins obscurément, en déterminent la dimension: devant, mais, et, en tant que, entre... Dans une telle perspective, la mobilité, l'allure et le rythme de l'existence ou de la pensée, et de la phénoménalité qu'elle interroge, seraient en revanche dits par car, donc, ou au contraire, par à, l'à de " je suis à toi », et, après, etc, qui disent une multiplicité, une transitivité et un inachèvement. 